\title{
Location of Farmers Warehouse at Adaklu Traditional Area, Volta Region, Ghana
}

\author{
Vincent Tulasi, ${ }^{1}$ Isaac Kwasi Adu, ${ }^{2}$ and Elikem Kofi Krampa ${ }^{1}$ \\ ${ }^{1}$ Department of Mathematics and Statistics, Ho Polytechnic, Ho, Ghana \\ ${ }^{2}$ Department of Mathematics, Valley View University, Techiman Campus, Techiman, Ghana \\ Correspondence should be addressed to Vincent Tulasi; vincentcockel@gmail.com
}

Received 2 December 2015; Revised 29 March 2016; Accepted 14 April 2016

Academic Editor: Manlio Gaudioso

Copyright (c) 2016 Vincent Tulasi et al. This is an open access article distributed under the Creative Commons Attribution License, which permits unrestricted use, distribution, and reproduction in any medium, provided the original work is properly cited.

\begin{abstract}
Postharvest loss is one major problem farmers in Adaklu Traditional Area that most Ghanaian farmers face. As a result, many farmers wallow in abject poverty. Warehouses are important facilities that help to reduce postharvest loss. In this research, Beresnev pseudo-Boolean Simple Plant Location Problem (SPLP) model is used to locate a warehouse at Adaklu Traditional Area, Volta Region, Ghana. This model was used because it gives a straightforward computation and produces no iteration as compared with other models. The SPLP is a problem of selecting a site from candidate sites to locate a plant so that customers can be supplied from the plant at a minimum cost. The model is made up of fixed cost and transportation cost. Location index ordering matrix was developed from the transportation cost matrix and we used it with the fixed cost and differences between variable costs to formulate the Beresnev function. Linear term developed from the function which was partial is pegged to obtain a complete solution. Of the 14 notable communities considered, Adaklu Waya is found most suitable for the setting of the warehouse. The total cost involved is GhC 78,180.00.
\end{abstract}

\section{Introduction}

Food crop production is the major occupation of the people of Adaklu. About $85 \%$ of the citizenry depend on these food crops for their well-being. Major food crops like maize, cassava, groundnut, and yam are produced.

The farmers largely depend on the natural rainfall for the cultivation of their crops. Food crops are cultivated annually. During bumper harvest, however, food crops are left exposed to bad weather and animals for destruction. Bushfire most of the time also destroys food crops stored in farm by farmers. Farm produce most of the time is stolen by thieves as there is no proper storage of the crops.

To prevent food crops going waste, most farmers do sell their food crops at very cheap prices, thereby making huge losses.

The objective of this study is to help locate a place where warehouse could be built to store farm produce. It is also to help farmers minimize cost of transporting farm produce to market centers. Warehouses are basically used for storing raw materials and finished goods $[1,2]$. They balance production schedule and demand, consolidate products from several different manufacturers and suppliers, and reduce inventory costs [3].

Quite a number of studies dealing with location of warehouses and similar facilities have been carried out in many different countries [3,4] using the Simple Plant Location Problem (SPLP) otherwise known as Uncapacitated Facility Location Problem (UFLP) [5-7]. The UFLP is the problem in which an arbitrary number of customers can be connected to a facility. If each facility has a limit on the number of customers it can serve, the problem becomes a capacitated facility location problem (CFLP) Ling-Yun et al. [1]; Cornuejols et al. [8]; and Tragantalerngsak et al. [9].

On problem of Mathematical Standardization Theory, Beresnev [10] developed Simple Plant Location Problem (SPLP) model which was used to locate site for building a plant. The model is made up of fixed cost and transportation cost. He developed location index ordering matrix from the transportation cost matrix and used it with the fixed cost and 


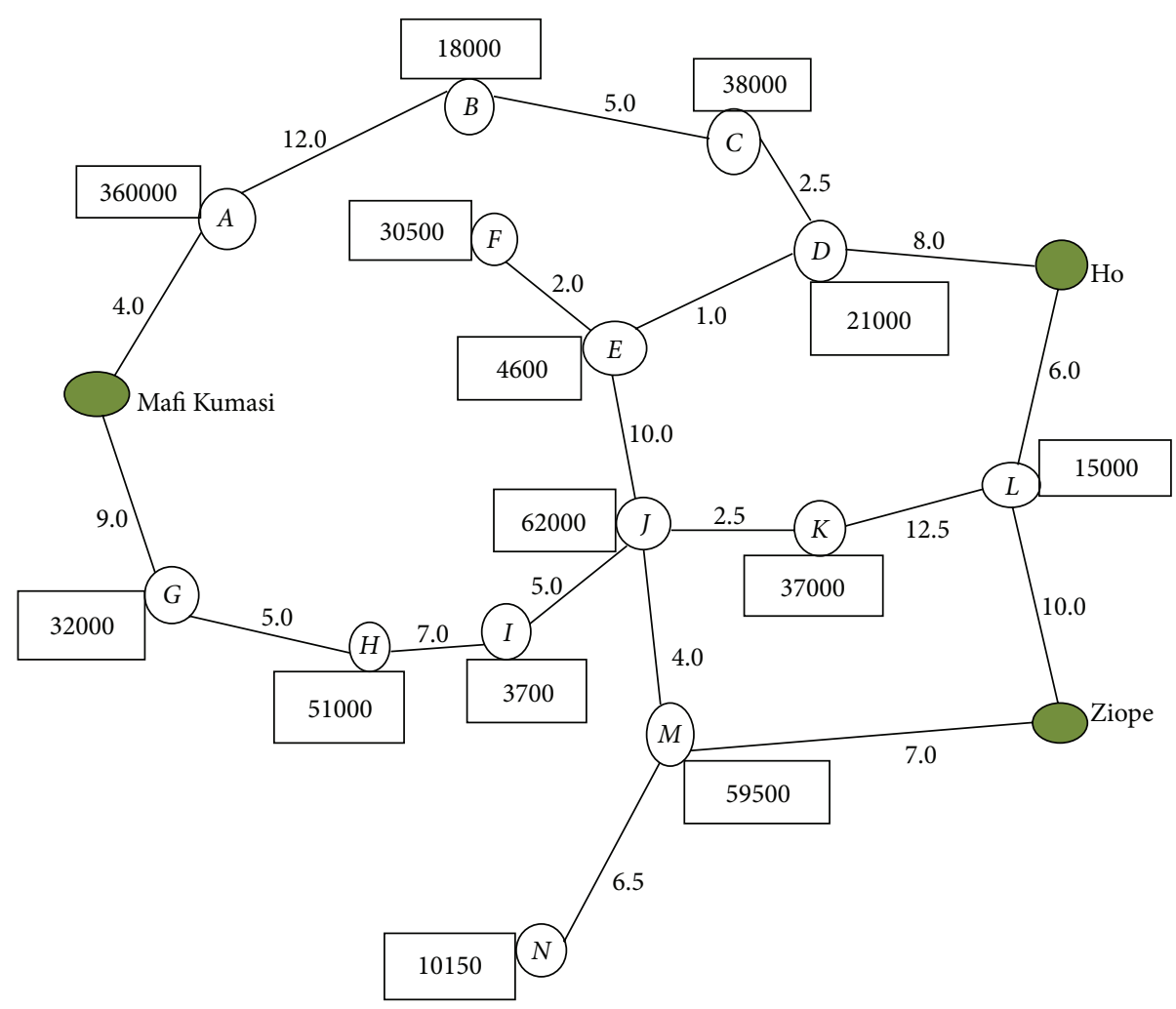

FIGURE 1: The figure shows the coded values of the fourteen communities with the distances linking them. The distances are shown on the arcs of the network and the fixed costs (in $\mathrm{GhC}$ ) of each community are indicated in the boxes.

differences between variable costs to formulate the Beresnev function.

Hajiaghayi et al. [11]; Hindi and Pieńkosz [12]; Fisher [13]; and Geoffrion and McBride [14] studied the UFLP with general setup cost functions. Their problem was motivated by an application in placing servers on the Internet. The setup cost of a server is a nondecreasing function of the number of clients connected to it. When the number of clients increases, the cost per client decreases since they share some common expenses. Therefore, the setup cost function exhibits concavity due to economies of scale. This problem was formulated as a generalized UFLP and a Mixed Integer Problem (MIP) was applied to solve the resulting model. They concluded that an efficient result was obtained.

\section{Research Methodology}

The data obtained for this project is from two main sources.

The first data is the distances between various (notable) towns in Adaklu Traditional Area. (refer to Appendix B). This data was obtained from the Department of Feeder Roads, Ho. The distances were measured in kilometers.
The second data deals with the estimated cost for establishing a modern warehouse in each community in the area. Masons, carpenters, and dealers in building materials in the communities were engaged in providing estimates for putting up the warehouse.

Adaklu Traditional Area is located at the eastern part of Ho, the Volta Regional Capital. It is made up of 46 small towns and villages with Adaklu Abuadi as its traditional capital. The area is not opened to commercial activities as the communities are widely dispersed and the road network is in a very deplorable state. Food crop farming is the major occupation for the people. Unfortunately, due to its inaccessibility and noncommercial nature, food crops obtained are spoilt over time as a result of postharvest losses (a sketch map of the traditional area is shown in Figure 2).

Fourteen notable communities (see Appendix C for the communities with their coded values) were selected for the project as shown in Figure 1.

2.1. Model Formulation. The Beresnev pseudo-Boolean SPLP is formulated as follows $[10,15]$ :

$$
\beta_{[F / C]}(Y)=\sum_{i=1}^{m} f_{i}\left(1-y_{i}\right)+\sum_{j=1}^{n}\left\{\Delta c[0, j]+\sum_{k=1}^{m-1} \Delta c[k, j] \cdot \prod_{r=1}^{k} y_{\varphi_{r_{j}}}\right\}
$$




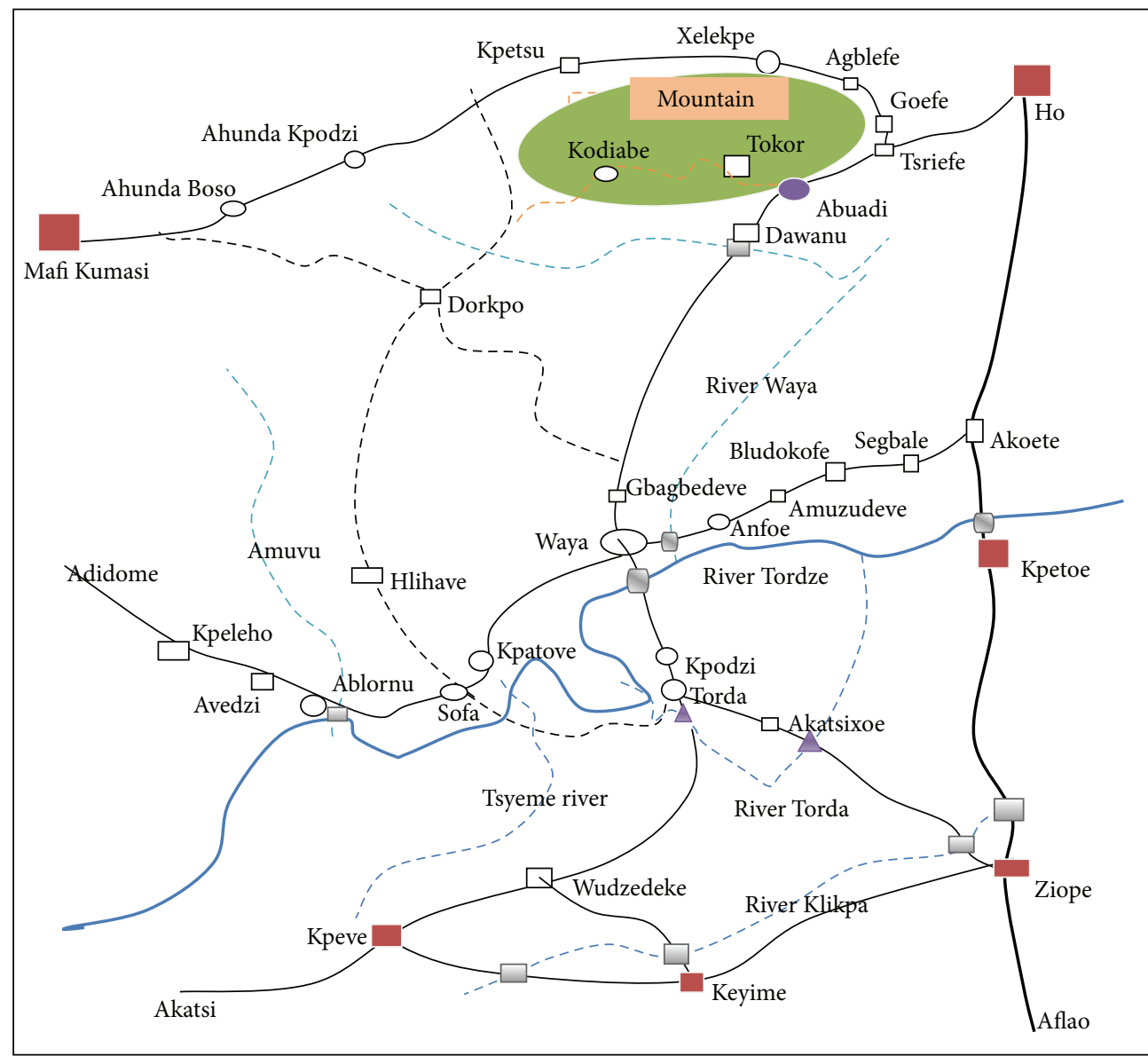

Towns not part of the traditional area

— First-class road

$\sim$ A third-class road, most vehicles can ply

$\sim$ Major river

... Footpath (only bicycles, motorbikes, and tractors can ply)

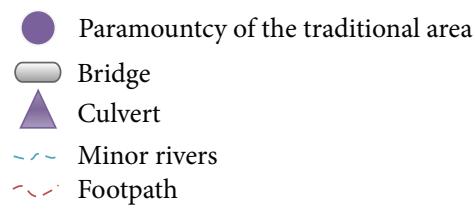

Figure 2

with the following conditions:

(a) if $a_{k} \geq 0$, then there is an optimal solution $y^{*}$ in which $a_{k}$ of $y^{*}$ is 0 , else

(b) if $a_{k}<0$ and $a_{k}+t_{k} \leq 0$ for each $k \in I$ in $\beta_{[F / C]}(Y)$ for the SPLP instance $[F / C]$, then there is solution $(1,1, \ldots, 1,0)$ assuming that the site indices are arranged in decreasing order of $f_{i}+\sum_{j \in J} c_{i j}$ values. That is, the optimal solution occurs at the minimum $a_{k}$, else

(c) if $a_{k} \in \mathscr{R}$ and $a_{k}+t_{k} \leq 0$, then there is an optimal solution $y^{*}$ in which $a_{k}$ of $y^{*}$ is 1 , provided that $y^{*} \neq 1$ for some $i \neq k$, where $a_{k}=\left(\sum_{j: \pi 1 j=k} \Delta c[1, j]\right)+f_{k}$ is the coefficient of the linear terms corresponding to $y_{k}$ and $t_{k}=\sum_{j ; k \in[\pi 1 j, \ldots, \pi p j]}^{n} j+1 \sum_{p=2}^{m-1} \Delta c[p, j]$ is the sum of the coefficients of all nonlinear terms containing $y_{k}$ for each site index $k$.

In (1), $A$ is the fixed cost, $B$ is the smallest number in each column of the transportation cost matrix, $C$ is the differences in the elements in each column of the transportation cost matrix, and $D$ is the products of the $y_{i}$ values that is maintained after computation of each column. $B, C$, and $D$ form the variable costs of the model.

\subsection{Algorithm for Computing Beresnev Pseudo-Boolean SPLP}

Step 1. Input edge matrix and the vector of facility setup cost (Appendix D).

Step 2. Do all pairs shortest path for the $14 \times 14$ transportation cost matrix $\left(C_{i j}\right)$ (Table 1$)$. 
TABLE 1: All pairs shortest path for edge matrix (see Appendix G) using MATLAB code.

\begin{tabular}{cccccccccccccccc}
\hline & $f_{i}$ & $A$ & $B$ & $C$ & $D$ & $E$ & $F$ & $G$ & $H$ & $I$ & $J$ & $K$ & $L$ & $M$ & $N$ \\
\hline$A$ & 3600 & 0.0 & 12.0 & 17.0 & 19.5 & 20.5 & 22.5 & 13.0 & 18.0 & 25.0 & 30.0 & 32.5 & 33.5 & 34.0 & 40.5 \\
$B$ & 1800 & 12.0 & 0.0 & 5.0 & 7.5 & 8.5 & 10.5 & 25.0 & 30.0 & 23.5 & 18.5 & 21.0 & 21.5 & 22.5 & 29.0 \\
$C$ & 3800 & 17.0 & 5.0 & 0.0 & 2.5 & 3.5 & 5.5 & 30.0 & 25.5 & 18.5 & 13.5 & 16.0 & 16.5 & 17.5 & 24.0 \\
$D$ & 2100 & 19.5 & 7.5 & 2.5 & 0.0 & 1.0 & 3.0 & 28.0 & 23.0 & 16.0 & 11.0 & 13.5 & 14.0 & 15.0 & 21.5 \\
$E$ & 4600 & 20.5 & 8.5 & 3.5 & 1.0 & 0.0 & 2.0 & 27.0 & 22.0 & 15.0 & 10.0 & 12.5 & 15.0 & 14.0 & 20.5 \\
$F$ & 3050 & 22.5 & 10.5 & 5.5 & 3.0 & 2.0 & 0.0 & 29.0 & 24.0 & 17.0 & 12.0 & 14.5 & 17.0 & 16.0 & 22.5 \\
$G$ & 3200 & 13.0 & 25.0 & 30.0 & 28.0 & 27.0 & 29.0 & 0.0 & 5.0 & 12.0 & 17.0 & 19.5 & 32.0 & 21.0 & 27.5 \\
$H$ & 5100 & 18.0 & 30.0 & 25.5 & 23.0 & 22.0 & 24.0 & 5.0 & 0.0 & 7.0 & 12.0 & 14.5 & 27.0 & 16.0 & 22.5 \\
$I$ & 3700 & 25.0 & 23.5 & 18.5 & 16.0 & 15.0 & 17.0 & 12.0 & 7.0 & 0.0 & 5.0 & 7.5 & 20.0 & 9.0 & 15.5 \\
$J$ & 6200 & 30.0 & 18.5 & 13.5 & 11.0 & 10.0 & 12.0 & 17.0 & 12.0 & 5.0 & 0.0 & 2.5 & 15.0 & 4.0 & 10.5 \\
$K$ & 3700 & 32.5 & 21.0 & 16.0 & 13.5 & 12.5 & 14.5 & 19.5 & 14.5 & 7.5 & 2.5 & 0.0 & 12.5 & 6.5 & 13.0 \\
$L$ & 1500 & 33.5 & 21.5 & 16.5 & 14.0 & 15.0 & 17.0 & 32.0 & 27.0 & 20.0 & 15.0 & 12.5 & 0.0 & 17.0 & 23.5 \\
$M$ & 5950 & 34.0 & 22.5 & 17.5 & 15.0 & 14.0 & 16.0 & 21.0 & 16.0 & 9.0 & 4.0 & 6.5 & 17.0 & 0.0 & 6.5 \\
$N$ & 1015 & 40.5 & 29.0 & 24.0 & 21.5 & 20.5 & 22.5 & 27.5 & 22.5 & 15.5 & 10.5 & 13.0 & 23.5 & 6.5 & 0.0 \\
\hline
\end{tabular}

Step 3. Find location index ordering matrix for the transportation cost matrix $\left(C_{i j}\right)$ using the formula $\omega_{j}=\left(\varphi_{1 j}, \ldots\right.$, $\left.\varphi_{14 j}\right)^{T}$ such that $c_{\varphi_{1 j}} j \leq c_{\varphi_{2 j}} j \leq \cdots \leq c_{\varphi_{14 j}} j$ (Appendix E).

Step 4. Compute differences between elements of each column in the transportation cost matrix using the formula $\Delta c[0, j]=c_{\varphi_{1 j}} j$, and $\Delta c[l, j]=c_{\varphi_{(l+1) j}} j-c_{\varphi_{1 j}} j, l=1, \ldots, 14$ (see (4)).

Step 5. Use the result in Step $4,(\Delta c[0, j])$ and $(\Delta c[l, j])$, and the setup cost function $\left(f_{i}\right)$ in Beresnev function (see (1)) to establish objective function.
Step 6. Compute coefficients of the linear term $a_{k}$ and the sum of the coefficients of the nonlinear terms $t_{k}$ in the objective function. Test for complete solution or partial solution using the conditions (a), (b), and (c) above. If complete solution occurs, stop and draw conclusion, else.

Step 7. Peg partial solution to obtain complete solution and then draw conclusion.

Equation (2) shows one of the twenty ordering matrices:

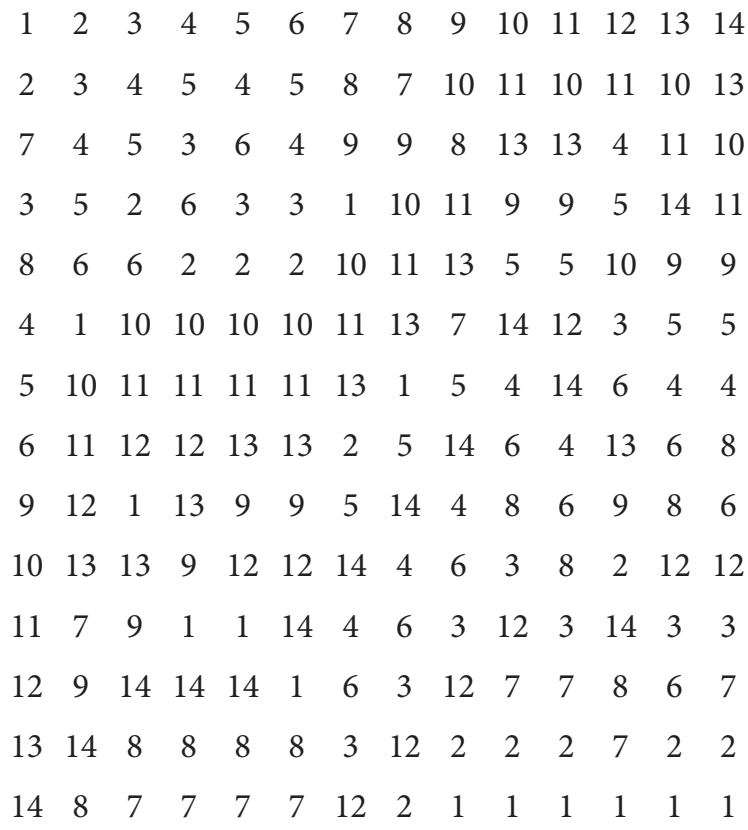


Equation (3) shows the arrangement of the transportation cost matrix using the ordering matrix in (2) (Appendix F):

$\begin{array}{cccccccccccccc}0 & 0 & 0 & 0 & 0 & 0 & 0 & 0 & 0 & 0 & 0 & 0 & 0 & 0 \\ 12 & 5.0 & 2.5 & 1.0 & 1.0 & 2.0 & 5.0 & 5.0 & 5.0 & 2.5 & 2.5 & 12.5 & 4.0 & 6.5 \\ 13 & 7.5 & 3.5 & 2.5 & 2.0 & 3.0 & 12 & 7.0 & 7.0 & 4.0 & 6.5 & 14 & 6.5 & 10.6 \\ 17 & 8.5 & 5.0 & 3.0 & 3.5 & 5.5 & 13 & 12 & 7.5 & 5.0 & 7.5 & 15 & 6.5 & 13 \\ 18 & 10.5 & 5.5 & 7.5 & 8.5 & 10.5 & 17 & 14.5 & 9.0 & 10 & 12.5 & 15 & 9.0 & 15.5 \\ 19.5 & 12 & 13.5 & 11 & 10 & 12 & 19.5 & 16 & 12 & 10.5 & 12.5 & 16.5 & 14 & 20.5 \\ 20.5 & 18.5 & 16 & 13.5 & 12.5 & 14.5 & 21 & 18 & 15 & 11 & 13 & 17 & 15 & 21.5 \\ 22.5 & 21 & 16.5 & 14 & 14 & 16 & 25 & 22 & 15.5 & 12 & 13.5 & 17 & 16 & 22.5 \\ 25 & 21.5 & 17 & 15 & 15 & 17 & 27 & 22.5 & 16 & 12 & 14.5 & 20 & 16 & 22.5 \\ 30 & 22.5 & 17.5 & 16 & 15 & 17 & 27.5 & 23 & 17 & 13.5 & 14.5 & 21.5 & 17 & 23 \\ 32.5 & 23.5 & 18.5 & 19.5 & 20.5 & 22.5 & 28 & 24 & 18.5 & 15 & 16 & 23.5 & 17.5 & 24 \\ 33.5 & 25 & 24 & 21.5 & 20.5 & 22.5 & 29 & 25.5 & 20 & 17 & 19.5 & 27 & 21 & 27.5 \\ 34 & 29 & 25.5 & 23 & 22 & 24 & 30 & 27 & 23.5 & 18.5 & 21 & 32 & 22.5 & 29.5 \\ 40.5 & 30 & 30 & 28 & 27 & 29 & 32 & 30 & 25 & 30 & 32.5 & 33.5 & 34 & 40.5\end{array}$

Equation (4) shows differences in the transportation cost matrix in (3):

$\begin{array}{cccccccccccccc}0 & 0 & 0 & 0 & 0 & 0 & 0 & 0 & 0 & 0 & 0 & 0 & 0 & 0 \\ 12 & 5.0 & 2.5 & 1.0 & 1.0 & 2.0 & 5.0 & 5.0 & 5.0 & 2.5 & 2.5 & 12.5 & 4.0 & 6.5 \\ 1.0 & 2.5 & 1.0 & 1.5 & 1.0 & 1.0 & 7.0 & 2.0 & 2.0 & 1.5 & 4.0 & 1.5 & 2.5 & 4.0 \\ 4.0 & 1.0 & 1.5 & 0.5 & 1.5 & 2.5 & 1.0 & 5.0 & 0.5 & 1.0 & 1.0 & 1.0 & 0.0 & 2.5 \\ 1.0 & 2.0 & 0.5 & 4.5 & 5.0 & 5.0 & 4.0 & 2.5 & 1.5 & 5.0 & 5.0 & 0.0 & 2.5 & 2.5 \\ 1.5 & 1.5 & 8.0 & 3.5 & 1.5 & 1.5 & 2.5 & 1.5 & 3.0 & 0.5 & 0.0 & 1.5 & 5.0 & 5.0 \\ 1.0 & 6.5 & 2.5 & 2.5 & 2.5 & 2.5 & 1.5 & 2.0 & 3.0 & 0.5 & 0.5 & 0.5 & 1.0 & 1.0 \\ 2.0 & 2.5 & 0.5 & 0.5 & 1.5 & 1.5 & 4.0 & 4.0 & 0.5 & 1.0 & 0.5 & 0.0 & 1.0 & 1.0 \\ 2.5 & 0.5 & 0.5 & 1.0 & 1.0 & 1.0 & 2.0 & 2.5 & 0.5 & 0.0 & 1.0 & 3.0 & 0.0 & 0.0 \\ 5.0 & 1.0 & 0.5 & 1.0 & 0.0 & 0.0 & 0.5 & 0.5 & 1.0 & 1.5 & 0.0 & 1.5 & 1.0 & 0.5 \\ 2.5 & 1.0 & 1.0 & 3.5 & 5.5 & 5.5 & 0.5 & 1.0 & 1.5 & 1.5 & 1.5 & 2.0 & 0.5 & 1.0 \\ 1.0 & 1.5 & 5.5 & 2.0 & 0.0 & 0.0 & 1.0 & 1.5 & 1.5 & 2.0 & 3.5 & 3.5 & 3.5 & 3.5 \\ 0.5 & 4.0 & 1.5 & 1.5 & 1.5 & 1.5 & 1.0 & 1.5 & 3.5 & 1.5 & 1.5 & 5.0 & 1.5 & 2.0 \\ 6.5 & 1.0 & 4.5 & 5.0 & 5.0 & 5.0 & 2.0 & 3.0 & 1.5 & 11.5 & 11.5 & 1.5 & 11.5 & 11\end{array}$

Now, the fixed cost of establishing the warehouse at each of the candidate sites $A$ to $N$ is

$Y=f_{i}=36000,1800,38000,21000,46000,30500,32000,51000,37000,62000,37000,15000,59500,10150$. 
Substituting the fixed cost and values in (4) into the Beresnev function yields

$$
\begin{aligned}
\beta_{[F / C]}, & \omega(Y) \\
= & 3600-3600 y_{1}+1800-1800 y_{2}+3800 \\
& -3800 y_{3}+\cdots \\
& +2 y_{11} y_{10} y_{13} y_{9} y_{12} y_{5} y_{14} y_{4} y_{6} y_{8} y_{3} y_{7} \\
& +11 y_{11} y_{10} y_{13} y_{9} y_{12} y_{5} y_{14} y_{4} y_{6} y_{8} y_{3} y_{7} y_{2} \\
= & 49315-3588 y_{1}-1795 y_{2}-3797.5 y_{3}-2099 y_{4} \\
& -4059 y_{5}-3048 y_{6}-2195 y_{7}-5095 y_{8} \\
& -3695 y_{9}-6197.5 y_{10}-3697.5 y_{11}-1487.5 y_{12} \\
& -5946 y_{13}-1008.5 y_{14}+1 y_{1} y_{2}+\cdots \\
& +2 y_{11} y_{10} y_{13} y_{9} y_{12} y_{5} y_{14} y_{4} y_{6} y_{8} y_{3} y_{7} \\
& +11 y_{11} y_{10} y_{13} y_{9} y_{12} y_{5} y_{14} y_{4} y_{6} y_{8} y_{3} y_{7} y_{2} .
\end{aligned}
$$

\section{Results and Discussions}

Computation of optimal site and objective function values is as follows.

We observe that the coefficients $a_{k}$ of the linear terms of $y_{i}$ in (6) for $i=1: 14$ are, respectively,

$$
\begin{aligned}
& -3588,-1795,-3797,-2099,-4059,-3048, \\
& -2195,-5095,-3695,-6197.5,-3697.5 \\
& -1487.5,-5946,-1008.5
\end{aligned}
$$

The sum $t_{k}$ of the coefficients of the nonlinear terms of $y_{i}$ is

$$
\begin{aligned}
& y_{1}=114, \\
& y_{2}=201, \\
& y_{3}=235, \\
& y_{4}=261, \\
& y_{5}=263, \\
& y_{6}=246.5, \\
& y_{7}=148, \\
& y_{8}=200.5, \\
& y_{9}=248, \\
& y_{10}=280.5, \\
& y_{11}=254, \\
& y_{12}=168.5, \\
& y_{13}=240.5, \\
& y_{14}=159 .
\end{aligned}
$$

TABLE 2: Result of (6).

\begin{tabular}{lccc}
\hline$y_{i}$ & $a_{k}$ & $t_{k}$ & $a_{k}+t_{k}$ \\
\hline$y_{1}$ & -3588 & 114 & -3474 \\
$y_{2}$ & -1795 & 201 & -1594 \\
$y_{3}$ & -3797 & 235 & -3562 \\
$y_{4}$ & -2099 & 261 & 1838 \\
$y_{5}$ & -4059 & 263 & 3796 \\
$y_{6}$ & -3048 & 246.5 & 2801.5 \\
$y_{7}$ & -2195 & 148 & 2047 \\
$y_{8}$ & -5095 & 200.5 & -4894.5 \\
$y_{9}$ & -3695 & 248 & 3447 \\
$y_{10}$ & -6197.5 & 280.5 & -5917 \\
$y_{11}$ & -3697.5 & 254 & -3442.5 \\
$y_{12}$ & -1487.5 & 168.5 & -1319 \\
$y_{13}$ & -5946 & 240.5 & -5705.5 \\
$y_{14}$ & -1008.5 & 159 & -849.5 \\
\hline
\end{tabular}

We can see that conditions (a) and (c) do not hold because $a_{k}<0 \forall k \in i$ in (6).

Condition (b) holds; that is, $a_{k}<0$ and $a_{k}+t_{k}<0$.

Now $a_{k}+t_{k}<0$ for each $k \in I$. Equation (6) is therefore a partial solution.

To obtain complete solution, we peg the coefficients $a_{k}$ of the $y_{i}$ which is done below.

(1) We arrange the coefficients $a_{k}$ of the linear term of $y_{i}$ in (6) in ascending order as follows: $-6197.5,-5946$, $-5095,-4059,-3797.5,-3697.5,-3695,-3588$, $-3048,-2195,-2099,-1795,-1487.5,-1008.5$.

(2) We assign the solutions $(0,1,1,1,1,1,1,1,1,1,1,1,1$, $1,1)$, respectively, to $y_{k}$ corresponding to coefficient $a_{k}$ in the arrangement above.

From $y_{i}=\{0$, if $i \in S ; 1$, otherwise $\}$ for each $i=1, \ldots$, 14 , the optimal solution therefore occurs at $y_{10}$.

For the cost, we substitute the solution $(0,1,1,1,1,1,1,1$, $1,1,1,1,1,1,1)$ into (6) and obtain the objective value:

$$
\begin{aligned}
\beta_{[F / C]} Y= & 49315-3588(1)-1795-3797.5-2099 \\
& -4059-3048-2195-5095-3695 \\
& -6197.5(0)-3697.5-1487.5-5946 \\
& -1008.5+1+4+1+1.5+1+2+2.5+5 \\
& +5+2.5+1+2+1.5+6.5+2.5+1+1.5 \\
& +0.5+8+1+1.5+0.5+4.5+3.5+1+1 \\
& +1.5+5+1.5+2+1+2.5+5+1.5+5 \\
& +7+1+4+5+2+5+5+2+1.5+2.5 \\
& +12.5+1.5+1+4+6.5+4+0+0+\cdots \\
& +0=493290-415110=78180
\end{aligned}
$$

We observe from Table 2 that $a_{k}<0$ and $a_{k}+t_{k}<0 \forall k \epsilon$ I. Equation (6) is therefore a partial solution. We peg the coefficients $a_{k}$ of $y_{k}$ in (6) to obtain complete solution. 
TABLE 3

(a)

\begin{tabular}{lccccccc}
\hline From & Waya & Anfoe & Waya & Torda & Kpetsu & Abuadi & Tserefe \\
\hline To & Torda & Akoete & Kpatove & Wudzedeke & Ahunda Boso & Tserefe & Xelekpe \\
\hline Distance $(\mathrm{km})$ & 4.0 & 12.5 & 5.0 & 6.5 & 12.0 & 1.0 & 2.5 \\
\hline
\end{tabular}

(b)

\begin{tabular}{lcccccc}
\hline From & Xelekpe & Waya & Kpatove & Ablornu & Kordiabe & Waya \\
\hline To & Kpetsu & Abuadi & Ablonu & Kpeleho & Abuadi & Anfoe \\
\hline Distance $(\mathrm{Km})$ & 5.0 & 10.0 & 7.0 & 5.0 & 2.0 & 2.5 \\
\hline
\end{tabular}

TABLE 4

(a)

\begin{tabular}{lccccccc}
\hline Codes & $A$ & $B$ & $C$ & $D$ & $E$ & $F$ & $G$ \\
\hline Communities & Ahunda Boso & Kpetsu & Xelekpe & Tserefe & Abuadi & Kordiabe & Kpeleho \\
\hline
\end{tabular}

(b)

\begin{tabular}{lccccccc}
\hline Codes & $H$ & $I$ & $J$ & $K$ & $L$ & $M$ & $N$ \\
\hline Communities & Ablornu & Kpatove & Waya & Anfoe & Akoete & Torda & Wudzedeke \\
\hline
\end{tabular}

We observe that the smallest $a_{k}$ of $y_{k}$ occurs at $y_{10}$. Node $J$ corresponds to $y_{10}$ which is Adaklu Waya.

Hence, by condition (c), the warehouse for the traditional area must be located at Adaklu Waya.

The total cost involved is GhC 78,180.00. This is made up of $\mathrm{GhC} 62,000.00$ facility setup cost and GhC 16,180.00 cost of transporting farm produce to the warehouse.

The Beresnev SPLP model is quicker and hence effective for the project. In view of the fact that the authors prefer Beresnev model to the other models, these other models were not used again in the modeling of the actual data.

\section{Conclusion}

The Beresnev pseudo-Boolean SPLP was used to model the data. It was used because it is a straightforward computation and it produces no iteration. The time used for obtaining the expected solution is also quicker.

From the analysis, of the 14 candidate sites considered for the location of the warehouse, Adaklu Waya is the most suitable site for the location of the warehouse.

The total cost involved is GhC 78,180.00. This amount is made up of GhC 62,000.00 fixed cost of setting up the warehouse and $\mathrm{GhC} 16,180.00$ transportation cost of collecting farm produce to the established warehouse. If each town was to build its own warehouse, there would be no transportation cost, but the total cost (fixed cost) to be incurred for the construction would be $\mathrm{GhC} 493,150.00$. This amount is the aggregation of the fixed costs of various sites. An amount of $\mathrm{GhC} 412,970.00$ would be saved by the traditional area if the warehouse is built at the selected location (Adaklu
Waya). Commercial activities would also boom in the area if collectively individual towns agreed and help to put up the warehouse at the selected site. Thus people would travel from far and near to patronize the warehouse especially in time of scarcity of food.

\section{Appendix}

\section{A. A Sketch Map of Adaklu Traditional Area}

See Figure 2.

\section{B. Distances between 14 Communities in Adaklu Traditional Area}

See Table 3.

\section{Coding of the Various Towns in Adaklu Traditional Area}

See Table 4.

\section{Edge Distance Matrix for the Network in Figure 1}

Equation (D.1) is a matrix in which links between two nodes are indicated by a real number while dash $(-)$ is used to indicate where there is no direct link (connection) between the nodes. The number 0 is used to indicate the connection of a node to itself: 


$$
C=\left[\begin{array}{ccccccccccccccc} 
& A & B & C & D & E & F & G & H & I & J & K & L & M & N \\
A & 0 & 12 & - & - & - & - & - & - & - & - & - & - & - & - \\
B & 12 & 0 & 5 & - & - & - & - & - & - & - & - & - & - & - \\
C & - & 5 & 0 & 2.5 & - & - & - & - & - & - & - & - & - & - \\
D & - & - & 2.5 & 0 & 1 & - & - & - & - & - & - & - & - & - \\
E & - & - & - & 1 & 0 & 2 & - & - & - & 10 & - & - & - & - \\
F & - & - & - & - & 2 & 0 & - & - & - & - & - & - & - & - \\
G & - & - & - & - & - & - & 0 & 5 & - & - & - & - & - & - \\
H & - & - & - & - & - & - & 5 & 0 & 7 & - & - & - & - & - \\
I & - & - & - & - & - & - & - & 7 & 0 & 5 & - & - & - & - \\
J & - & - & - & - & 10 & - & - & - & 5 & 0 & 2.5 & - & 4 & - \\
K & - & - & - & - & - & - & - & - & 2.5 & 0 & 12.5 & - & - \\
L & - & - & - & - & - & - & - & - & - & 12.5 & 0 & - & - \\
M & - & - & - & - & - & - & - & - & 4 & - & - & 0 & 6.5 \\
N & - & - & - & - & - & - & - & - & - & - & - & - & 6.5 & 0
\end{array}\right] .
$$

\section{E. Computation of Arrangement of} Elements of (3) Using the Formula

$\Delta c[l, j]=c_{\varphi_{(l+1) j}} j-c_{\varphi_{1 j}} j, l=1: 14$

Consider

$$
\begin{aligned}
& c_{\varphi_{11}} j=c_{\varphi_{111}}=c_{\varphi_{11}}=0, \\
& c_{\varphi_{21}} j=c_{\varphi_{211}}=c_{\varphi_{21}}=12, \\
& c_{\varphi_{31}} j=c_{\varphi_{311}}=c_{\varphi_{71}}=13, \\
& c_{\varphi_{41}} j=c_{\varphi_{411}}=c_{\varphi_{31}}=17, \\
& c_{\varphi_{51}} j=c_{\varphi_{511}}=c_{\varphi_{P_{1}}}=18, \\
& c_{\varphi_{61}} j=c_{\varphi_{611}}=c_{\varphi_{41}}=19.5, \\
& c_{\varphi_{71}} j=c_{\varphi_{711}}=c_{\varphi_{51}}=20.5, \\
& c_{\varphi_{81}} j=c_{\varphi_{811}}=c_{\varphi_{61}}=22.5, \\
& c_{\varphi_{91}} j=c_{\varphi_{911}}=c_{\varphi_{91}}=25, \\
& c_{\varphi_{101}} j=c_{\varphi_{1011}}=c_{\varphi_{101}}=30, \\
& c_{\varphi_{111}} j=c_{\varphi_{111}}=c_{\varphi_{111}}=32.5, \\
& c_{\varphi_{121}} j=c_{\varphi_{1211}}=c_{\varphi_{121}}=33.5, \\
& c_{\varphi_{131}} j=c_{\varphi_{1311}}=c_{\varphi_{131}}=34, \\
& c_{\varphi_{141}} j=c_{\varphi_{1411}}=c_{\varphi_{141}}=40.5, \\
& c_{\varphi_{12}} j=c_{\varphi_{122}}=c_{\varphi_{22}}=0, \\
& c_{\varphi_{22}} j=c_{\varphi_{222}}=c_{\varphi_{32}}=5,
\end{aligned}
$$$$
c_{\varphi_{32}} j=c_{\varphi_{322}}=c_{\varphi_{42}}=7.5,
$$$$
c_{\varphi_{42}} j=c_{\varphi_{422}}=c_{\varphi_{52}}=8.5 \text {, }
$$$$
c_{\varphi_{52}} j=c_{\varphi_{522}}=c_{\varphi_{62}}=10.5 \text {, }
$$$$
c_{\varphi_{62}} j=c_{\varphi_{622}}=c_{\varphi_{12}}=12 \text {, }
$$$$
c_{\varphi_{72}} j=c_{\varphi_{722}}=c_{\varphi_{102}}=18.5 \text {, }
$$$$
c_{\varphi_{82}} j=c_{\varphi_{822}}=c_{\varphi_{112}}=21 \text {, }
$$$$
c_{\varphi_{92}} j=c_{\varphi_{922}}=c_{\varphi_{122}}=21.5 \text {, }
$$$$
c_{\varphi_{102}} j=c_{\varphi_{1022}}=c_{\varphi_{132}}=22.5 \text {, }
$$$$
c_{\varphi_{112}} j=c_{\varphi_{1122}}=c_{\varphi_{72}}=23.5 \text {, }
$$$$
c_{\varphi_{122}} j=c_{\varphi_{1222}}=c_{\varphi_{92}}=25 \text {, }
$$$$
c_{\varphi_{132}} j=c_{\varphi_{1322}}=c_{\varphi_{142}}=29 \text {, }
$$$$
c_{\varphi_{142}} j=c_{\varphi_{1422}}=c_{\varphi_{82}}=30 \text {, }
$$$$
c_{\varphi_{13}} j=c_{\varphi_{133}}=c_{\varphi_{33}}=0 \text {, }
$$$$
c_{\varphi_{23}} j=c_{\varphi_{233}}=c_{\varphi_{43}}=2.5 \text {, }
$$$$
c_{\varphi_{33}} j=c_{\varphi_{333}}=c_{\varphi_{53}}=3.5 \text {, }
$$$$
c_{\varphi_{43}} j=c_{\varphi_{433}}=c_{\varphi_{23}}=5 \text {, }
$$$$
c_{\varphi_{53}} j=c_{\varphi_{533}}=c_{\varphi_{63}}=5.5 \text {, }
$$$$
c_{\varphi_{63}} j=c_{\varphi_{633}}=c_{\varphi_{103}}=13.5 \text {, }
$$$$
c_{\varphi_{73}} j=c_{\varphi_{733}}=c_{\varphi_{113}}=16 \text {, }
$$ 
function $\operatorname{lip}(\mathrm{a})$

$\%$ to arrange numbers in ascending order $\mathrm{n}=$ length $(\mathrm{a})$;

for $\mathrm{j}=\mathrm{n}:-1: 1$

for $\mathrm{i}=1: \mathrm{j}-1$

if $\mathrm{a}(\mathrm{i})>\mathrm{a}(\mathrm{i}+1)$

$\mathrm{a}(\mathrm{i})=\mathrm{a}(\mathrm{i}+1)$; $\mathrm{p}=\mathrm{a}(\mathrm{i})$;

$\mathrm{a}(\mathrm{i}+1)=\mathrm{p}$;

end

end

end

$\operatorname{disp}(\mathrm{a})$

\section{G. MATLAB Code for All Pairs Shortest Path for Transportation Cost Matrix}

See Code 2.

\section{Additional Points}

(1) Further research on the topic should examine how the method could be employed to absolutely locate a single plant for communities. (2) The result of this research finding should be implemented by the district assembly and opinion leaders in the area.

\section{Competing Interests}

The authors declare that they have no competing interests.

\section{References}

$$
\begin{aligned}
& \text { \% input transportation cost matrix c } \\
& \text { functiongoslow }(G) \\
& {[n, n]=\operatorname{size}(G) \text {; }} \\
& \text { for } k=1: n \\
& \text { for } i=1: n \\
& \text { for } j=1: n \\
& \text { if } i==j \\
& G(i, j)=0 ; \\
& \text { elseif } G(i, j)==\inf \\
& \text { end } G(i, j)=\min (G(i, j), G(i, k)+G(k, j)) \\
& \text { end } \\
& \text { end } \\
& \text { end } \\
& \text { end }
\end{aligned}
$$

$$
\begin{gathered}
c_{\varphi_{83}} j=c_{\varphi_{833}}=c_{\varphi_{123}}=16.5, \\
c_{\varphi_{93}} j=c_{\varphi_{933}}=c_{\varphi_{13}}=17, \\
c_{\varphi_{103}} j=c_{\varphi_{1033}}=c_{\varphi_{133}}=17.5, \\
c_{\varphi_{113}} j=c_{\varphi_{1133}}=c_{\varphi_{93}}=18.5, \\
c_{\varphi_{123}} j=c_{\varphi_{1233}}=c_{\varphi_{143}}=24, \\
c_{\varphi_{133}} j=c_{\varphi_{1333}}=c_{\varphi_{83}}=25.5, \\
c_{\varphi_{143}} j=c_{\varphi_{1433}}=c_{\varphi_{73}}=30 .
\end{gathered}
$$

\section{F. MATLAB Code for Arranging Elements in the Column of Transportation Cost Matrix in Ascending Order}

See Code 1.
[1] W. Ling-Yun, Z. Xiang-Sun, and Z. Ju-Liang, Capacitated Facility Location with General Setup Cost, Institute of Applied Mathematics, Academy of Mathematics and Systems Science, Chinese Academy of Science, Beijing, China, 2006.

[2] B. F. AlMohammad, B. Goldengorin, D. Ghosh, and G. Sierksma, "Equivalent instances of the simple plant location problem," SOM Research Report 00A54, University of Groningen, Groningen, The Netherlands, 2000.

[3] A. Marín and B. Pelegrín, "The return plant location problem: modelling and resolution," European Journal of Operational Research, vol. 104, no. 2, pp. 375-392, 1998.

[4] S. Mehmet and R. G. Ali, A Discrete Particle Swarm Optimization Algorithm for Uncapacitated Facility Location Problem, Department of Industrial Engineering, Faculty of Engineering, Fatih University, Istanbul, Turkey, 2007.

[5] K. Aardal, M. Labbé, J. Leung, and M. Queyranne, On the TwoLevel Uncapacitated Facility Location Problem, Department of Computer Science, Utretcht University, TB Utrecht, The Netherlands, 1995.

[6] C. Beltran-Royo, J.-Ph. Vial, and A. Alonso-Ayuso, Solving the Uncapacitated Facility Location Problem with Semi-Lagrangian Relaxation, Statistics and Operation Research, Rey Juan Carlos University, Madrid, Spain, 2001.

[7] G. Ghiani, F. Guerriero, and R. Musmanno, "The capacitated plant location problem with multiple facilities in the same site," Computers \& Operations Research, vol. 29, no. 13, pp. 1903-1912, 2002.

[8] G. Cornuejols, R. Sridharan, and J. M. Thizy, "A comparison of heuristics and relaxations for the capacitated plant location problem," European Journal of Operational Research, vol. 50, no. 3, pp. 280-297, 1991.

[9] S. Tragantalerngsak, J. Holt, and M. Rönnqvist, "Lagrangian heuristics for the two-echelon, single-source, capacitated facility location problem," European Journal of Operational Research, vol. 102, no. 3, pp. 611-625, 1997.

[10] V. L. Beresnev, "On a problem of mathematical standardization theory," Upravliajemyje Sistemy, vol. 11, pp. 43-54, 1973.

[11] M. T. Hajiaghayi, M. Mahdian, and V. S. Mirrokni, "The facility location problem with general cost function," Working Paper, Massachusetts Institute of Technology, 2003. 
[12] K. S. Hindi and K. Pieńkosz, "Efficient solution of large scale, single-source, capacitated plant location problems," Journal of the Operational Research Society, vol. 50, no. 2-3, pp. 268-274, 1999.

[13] M. L. Fisher, "The lagrangian relaxation method for solving integer programming problems," Management Science, vol. 27, no. 1, pp. 1-18, 1981.

[14] A. M. Geoffrion and R. McBride, "Lagrangian relaxation applied to capacitated facility location problems," AIIE Transactions, vol. 10, no. 1, pp. 40-47, 1978.

[15] B. Goldengorin, G. Sierksma, G. A. Tijssen, and M. Tso, "The data-correcting algorithm for the minimization of supermodular functions," Management Science, vol. 45, no. 11, pp. 1539-1551, 1999. 


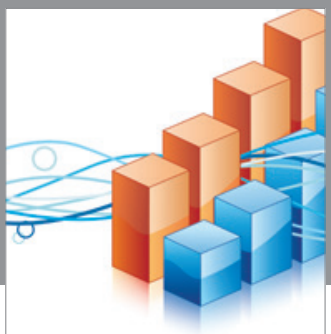

Advances in

Operations Research

vatem alat4

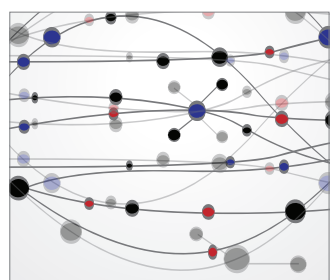

\section{The Scientific} World Journal
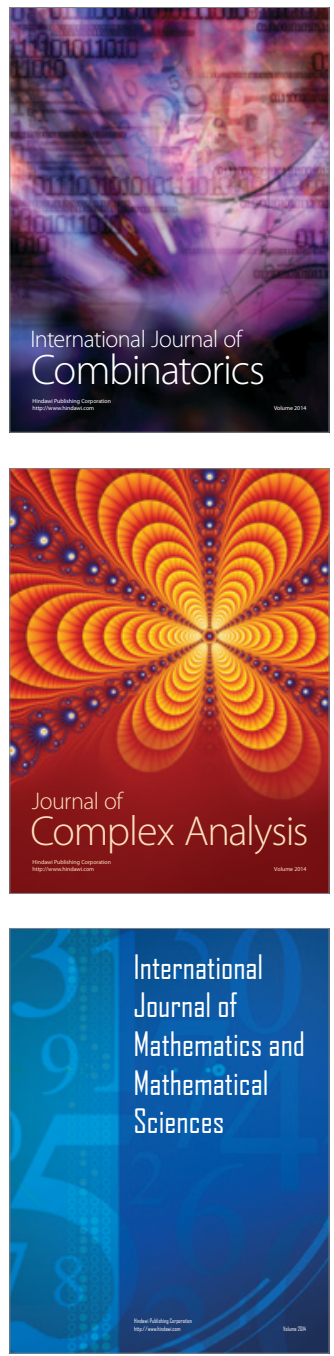
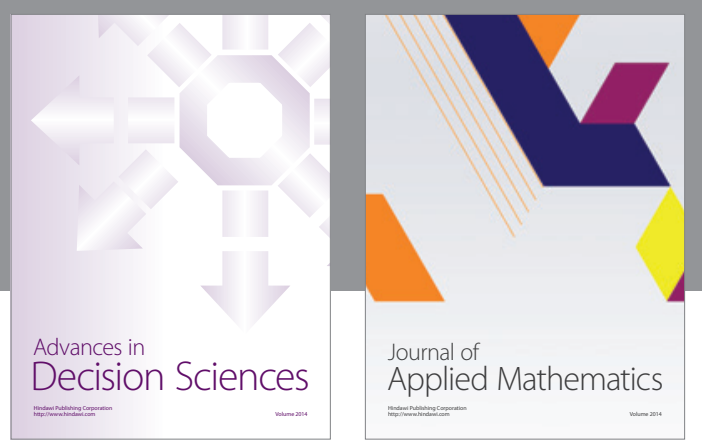

Algebra

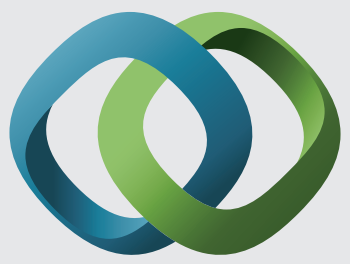

\section{Hindawi}

Submit your manuscripts at

http://www.hindawi.com
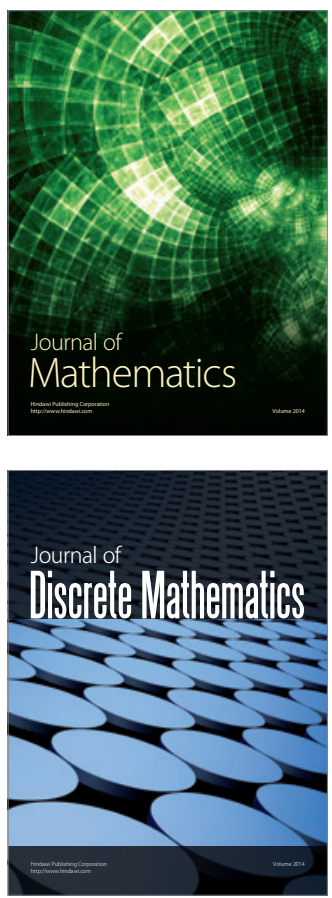

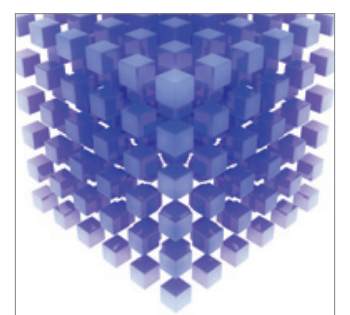

Mathematical Problems in Engineering
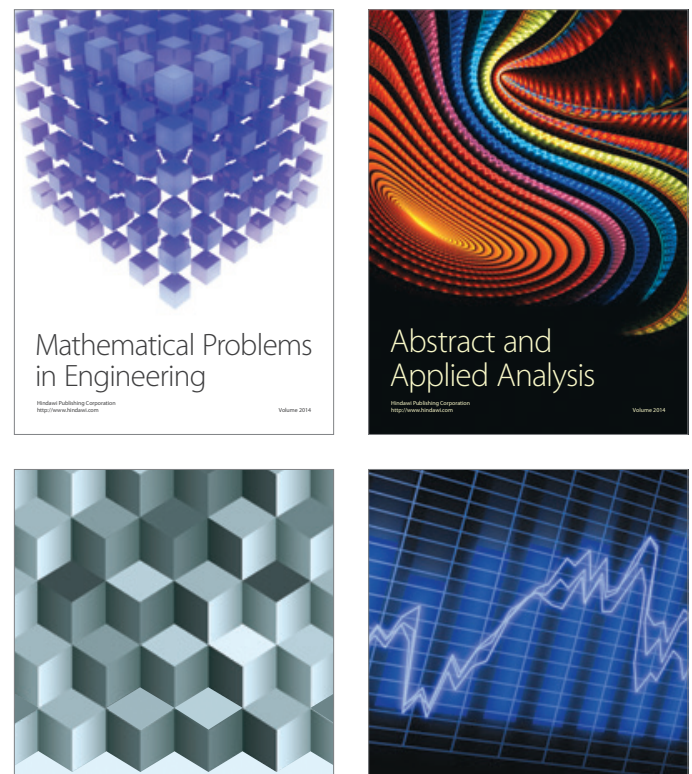

Journal of

Function Spaces

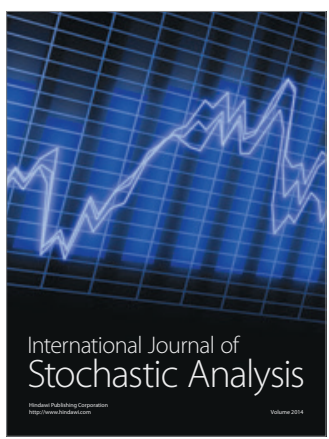

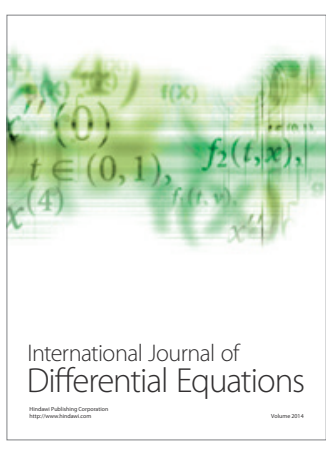
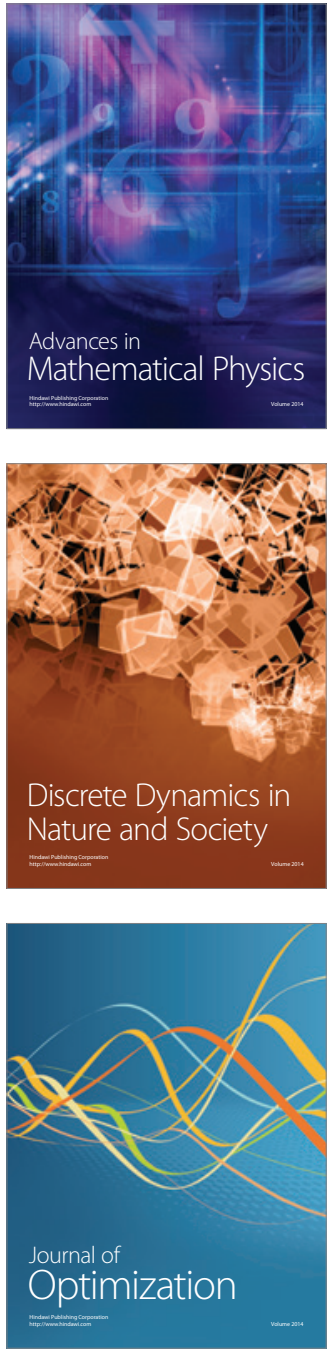